\title{
IS06
}

The Role of Cache-Centric Storage in Petascale and Exascale Deployments

\section{Vildibill* (DDN)}

\section{SUMMARY}

Existing parallel file systems are set to run into scalability roadblocks as HPC compute complexes grow.

DDN Testing and demonstrations presented at SC'13 in the US clearly showed the potential of cachecentric storage in alleviating the most serious of these roadblocks - the acute scaling hurdles of serial elements of parallel systems.

In this paper, Mike Vildibill will discuss how cache-centric storage approaches, including burst buffer technology, can be practically applied to resolve large scale computing IO bottlenecks from today through early exascale implementations. 